\title{
Numerical Simulation of Deck Waves of Container Vessel Based on Numerical Viscous Wave Tank
}

\author{
Yi Liu , Renqing Zhu, Lefei Li, Yu Zhang, Miao Xia
}

\begin{abstract}
When a vessel is sailing in rough wave, the large relative movement between the structure and the waves will cause the waves to flood the deck. Deck waves will cause damage to the deck equipment and destroy the integrity of the structure. This condition will not only lead to the loss of economic property, but will also cause serious casualty. The waves on the deck will cause a huge slamming load on the vessel, causing the structure of the bow deck to be damaged by the impact of the water and even causing the entire hull to sink. Therefore, it is very important to analysis the deck wave and study the wave law on the bow deck and structure on the upper deck. When we design civilian or military ships, deck wave is an important factor to structure of vessel, personnel safety and ship performance. In the paper, the container ship is modelled by CAD software and the mesh of wave tank is generated by HEXPRESS function, which is based on Fine/Marine's adaptive mesh generation technology. In addition to this, numerical model simulates the motion response of the container ship under different wave amplitude and vary ship's speed in regular wave. Moreover, the variation law of the wave phenomenon on the deck is analysed. Through this research, it is found that the waves on the deck will have a severe impact on the vessel's deck and the equipment on the upper deck. The well-designed wave-stopping structure can effectively reduce the impact of the water body on the structure.
\end{abstract}

Index Terms - Numerical viscous wave tank; Motion response; Regular wave; Fine/Marine; Deck waves

\section{INTRODUCTION}

At the end of the 19th century, some scholars discovered that the wave on the deck may have a certain impact on ship performance. The first systematic analysis of this phenomenon was Newton[1]. In 1959, Newton conducted a model test on a destroyer under regular and positive wave conditions. Apart from this, he computed the deck waves in the case of five different bow shapes. However, the experiment did not further explain at the theoretical level.

As the rapid development of computing technology and high performance computers in recent years, some numerical methods such as MAC, VOF, LEVEL-SET, SPH, CIP, which can accurately track reconstructed free surfaces, have been introduced to solve the problem of large deformation of free surface. And some of the calculation methods and techniques have been developed into mature CFD software. Applying the

software to the deck waves creates conditions for numerical simulation of the wave problem.

Yi Liu , School of Naval Architecture \& Ocean Engineering, Jiangsu University of Science and Technology, Zhenjiang, Jiangsu, China Renqing Zhu International College, Jiangsu University of Science and Technology, Zhenjiang, Jiangsu, China

Lefei Li, School of Naval Architecture \& Ocean Engineering, Jiangsu University of Science and Technology, Zhenjiang, Jiangsu, China

Yu Zhang, Taikai Automation Company Limited, Taian, Shandong, China

Miao Xia, School of Naval Architecture \& Ocean Engineering, Jiangsu University of Science and Technology, Zhenjiang, Jiangsu, China
Nielsen[2] used the N-S equation solver and the VOF method to perform two-dimensional and three- -dimensional numerical simulations of the deck waves during stationary and moving vessel. The motion of the hull (in the vertical direction only) is pre-calculated using the linear wave theory, and the transfer function is used to calculate the vertical relative motion of the ship's side caused by pitch and heave.

$\mathrm{Hu}[3]$ used the CIP method of tracking free faces to simulate hull motion and deck waves in waves in real time under laminar flow assumptions. This method describes the strong nonlinear characteristics of the floating body movement to a certain extent, and reflects the influence of the upper waves on the movement of the floating body. However, the study only predicts the motion response of the box under the action of regular waves in the two-dimensional theory, and does not consider the influence on the three-dimensional theory.

Rik[4] studied the sloshing of the tank and the wave climbing of the semi-submersible platform by solving the compressible $\mathrm{N}-\mathrm{S}$ equation and the VOF method to capture the free surface. The accurate slamming pressure results were obtained by this method. At the same time, the authors pointed out that considering the compressibility of air can make the numerical simulation closer to the physical reality and get more accurate slamming pressure. In addition, when the free surface changes drastically (such as the tank sloshing), the air is often mixed in the vicinity of the free surface, considering the compressibility of the gas, the simulated free surface can be made closer to the physical reality.

Bowen Shi[5] based on the CFD method to establish a three-dimensional viscous flow numerical wave tank, using the boundary condition wave method to generate the wave environment based on WaMoS-II measurement, and using the VOF method to capture the free surface. On this basis, the numerical simulation of the deck waves when the ship is sailing at different wave angles under high sea conditions is realized. Through analysis, the frequency of the deck waves under different wave angles of the DTMB5512 ship model is predicted by numerical calculation.

Guanghua He [6] based on the CIP method, established a time domain analysis model for the strong nonlinear motion response of ships in waves. In view of the viscous flow theory, the model solves the $\mathrm{N}-\mathrm{S}$ equation and considers the viscous effect and strong nonlinear effects of the ship's motion response and free-surface deformation. The strong nonlinear motion response model of vessel is programmed by FORTRAN, and has good convergence and accuracy, which is in good agreement with other numerical calculation results. In this paper, the motion response of the container ship and the wave on the deck under different vessel speeds and different amplitudes of waves are studied. The main contents are as follows: 
1. The basic theory of numerical simulation using CFD is described in detail.

2. Based on the viscous flow theory, a three dimensional numerical wave tank with wave-making and wave eliminating functions is established, and the wave tank is used to simulate the regular wave.

3. Select the 14000 TEU container vessel model to calculate the heave and pitch motion under a certain condition and compare it with the numerical results in other CFD software.

4. Calculate the motion response and deck wave calculations of container ships under different sea conditions and analysis the load changes of the deck waves on the bow deck and baffles.

\section{NUMERICAL MODEL}

\section{A. Control Model}

For viscous incompressible fluid, the N-S equation is simplified to:

$$
\frac{\partial u_{i}}{\partial t}+\frac{\partial}{\partial x_{j}}\left(u_{i} u_{j}\right)=-\frac{1}{\rho} \frac{\partial p}{\partial x_{j}}+v \frac{\partial}{\partial x_{j}}\left(\frac{\partial u_{i}}{\partial x_{j}}+\frac{\partial u_{j}}{\partial x_{i}}\right)
$$

Where: $x_{i}$ represents the coordinates, and $u_{i}$ indicates the velocity component in three directions.

Based on the Reynolds averaging method, and adding the SST $k-\omega$ model, the RANS equation is closed. Due to this model has lateral derivative terms and turbulent shear stress transport characteristics, it is widely used to the calculation of flows with back-pressure gradient.

The transport equation for the SST $k-\omega$ model

$$
\begin{aligned}
& \text { is: } \frac{\partial}{\partial t}(\rho k)+\frac{\partial}{\partial x_{i}}\left(\rho k u_{i}\right)=\frac{\partial}{\partial x_{j}}\left(\Gamma_{k} \frac{\partial k}{\partial x_{j}}\right)+\tilde{G}_{k}+Y_{k}+S_{k} \\
& \frac{\partial}{\partial t}(\rho \omega)+\frac{\partial}{\partial x_{i}}\left(\rho \omega u_{i}\right)=\frac{\partial}{\partial x_{j}}\left(\Gamma_{\omega} \frac{\partial \omega}{\partial x_{j}}\right)+G_{\omega}+D_{\omega}+S_{\omega}
\end{aligned}
$$

Where: $i, j$ the range of values is $(1,2,3), \tilde{G}_{k}$ is a term generated by the turbulent kinetic energy $(k)$ caused by the average velocity, $G_{\omega}$ is term of $\omega . \Gamma_{k}$ and $\Gamma_{\omega}$ are effective diffusion terms of $k$ and $\omega$ respectively. $D_{\omega}$ is a cross diffusion item, and are custom source items.

\section{B. Numerical Wave Theory}

The static water surface will fluctuate and leave the equilibrium position due to the action of wind or other external force. Gravity drives the liquid level to return to the horizontal level. Under the joint action of inertial force and gravity, the liquid surface reciprocates back and forth on the free surface to form a wave. The wave simulated in this paper is a linear small amplitude wave. For a finite water depth plane linear wave, the solution of the equation is as follows. Wave equation:

$$
\eta=\frac{H}{2} \cos (k x-\omega t)
$$

Speed potential:

$$
\phi=\frac{g H}{2 \omega} \frac{\cosh (z+d)}{\cosh k d} \sin (k x-\omega t)
$$

The dispersion relation between wave number $k$ and circle frequency $\omega$ :

$$
\frac{\omega^{2}}{g}=k \tanh k d
$$

The relationship between wavelength $\lambda$ and wave period T:

$$
\lambda=\frac{g}{2 \pi} T^{2} k \tanh \frac{2 \pi}{\lambda} d
$$

Speed in the $\mathrm{x}$ direction:

$$
u_{x}=\frac{g H K}{2 \omega} \frac{\cosh (z+d)}{\cosh k d} \cos (k x-\omega t)
$$

Speed in the $\mathrm{z}$ direction:

$$
u_{z}=\frac{g H K}{2 \omega} \frac{\sinh (z+d)}{\cosh k d} \sin (k x-\omega t)
$$

Where: Where: $\mathrm{H}$ is the wave height, $k$ is the wave number, $\omega$ is the wave circle frequency, g is the gravity acceleration, $\mathrm{d}$ is the static water depth, the $\mathrm{x}$-axis positive direction is the wave propagation direction, and the $\mathrm{z}$-axis is the vertical

\begin{tabular}{|c|c|c|c|}
\hline Property & Symbol & Unit & Value \\
\hline Total length & $\mathrm{L}_{\mathrm{OA}}$ & $\mathrm{m}$ & 369.59 \\
\hline $\begin{array}{ll}\text { Length } & \text { between } \\
\text { Perpendiculars } & \end{array}$ & $\mathrm{L}_{\mathrm{pp}}$ & $\mathrm{m}$ & 351 \\
\hline Breadth & B & $\mathrm{m}$ & 49 \\
\hline Depth & $\mathrm{D}$ & $\mathrm{m}$ & 30 \\
\hline Bow Draft & da & $\mathrm{m}$ & 14 \\
\hline End Draft & df & $\mathrm{m}$ & 14 \\
\hline Draft & $\mathrm{d}$ & $\mathrm{m}$ & 14 \\
\hline Volume of Displacement & $\nabla$ & $\mathrm{M}^{3}$ & 160027 \\
\hline $\begin{array}{l}\text { Longitudinal Position of } \\
\text { Gravity }\end{array}$ & $\mathrm{L}_{\mathrm{CG}}$ & $\mathrm{m}$ & 169.38 \\
\hline $\begin{array}{l}\text { Vertical Position of } \\
\text { Gravity }\end{array}$ & $\mathrm{V}_{\mathrm{CG}}$ & $\mathrm{m}$ & 19.46 \\
\hline $\begin{array}{l}\text { Longitudinal Radius of } \\
\text { Inertia }\end{array}$ & $\mathrm{K}_{\mathrm{yy}}$ & $\mathrm{m}$ & 87.7 \\
\hline $\begin{array}{l}\text { Transverse Radius of } \\
\text { Inertia }\end{array}$ & $\mathrm{K}_{\mathrm{xx}}$ & $\mathrm{m}$ & 17.2 \\
\hline
\end{tabular}
coordinate (the upward direction is positive), $\mathrm{z}=0$ means the water surface.

\section{The Container Model}

The vessel type used in this paper is 14000TEU container ship. The ship type and its parameters used in the simulation are shown in Table.1. The scale ratio used in the model is 1:75. The profile is shown in Fig.1. The model is shown in Fig.2.

Table. 1 the Principal particulars of 14000TEU

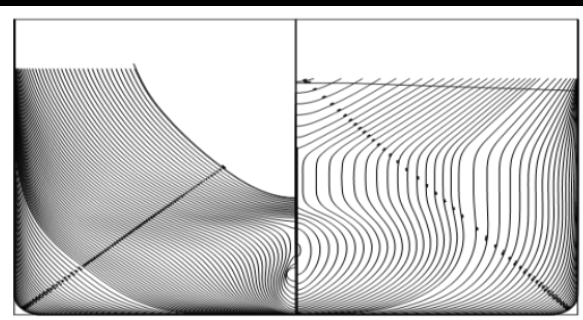

Fig.1 Ship Lines of 14000TEU Container Ship

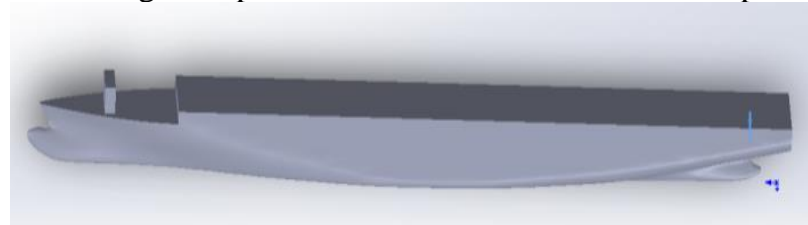

Fig.2 Geometry Model of 14000TEU Container Ship 


\section{Computational Grid and Monitoring Points}

The Fig. 3 shows the calculation grid of the container ship. The number of grids is $2.4^{*} 10^{\wedge} 6$. The calculation domain size is: the tank is $6_{\mathrm{Lpp}}$ in the direction of the ship, ${ }^{2} \mathrm{Lpp}$ in the ship width direction, and $1.2 \mathrm{Lpp}$ in the draft direction. In order to ensure the stability of the numerical simulation, the ship gradually accelerates from the stationary state to the speed $U$ by $1 / 4$ sinusoidal change, and the square calculation domain is selected during the FINE/Marine calculation process. Due to the symmetry of the calculated problem and the shape of the hull, in order to decrease the calculation time, only half of the hull and calculation domain are used in the calculation process.

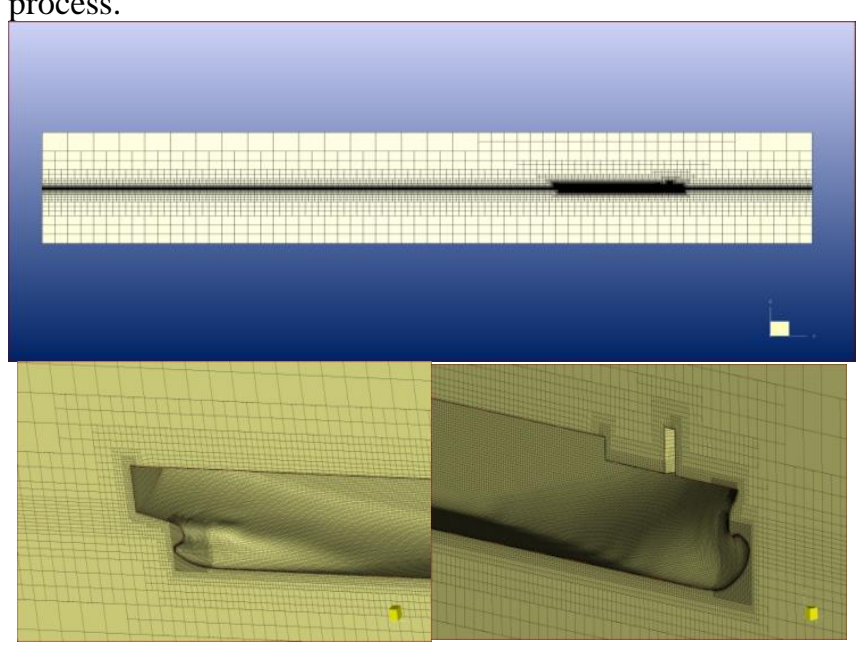

Fig.3 Computational Mesh of Vessel

In order to monitor the pressure load on the bow deck and the impact load on the baffle, this paper sets the pressure monitoring points on the bow deck and the baffle, and selects the comparative pressure points P19, P20. P28, P29 for analysis. The set position of the pressure point is shown in Fig.4 and Fig.5.

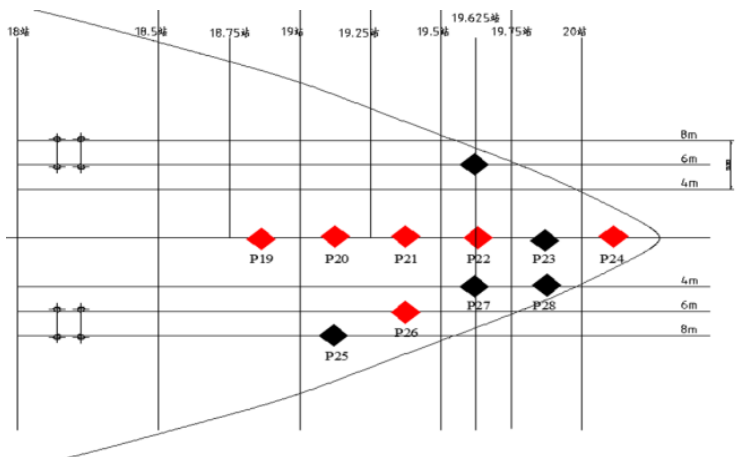

Fig.4 Pressure Monitoring Point on the Bow Deck

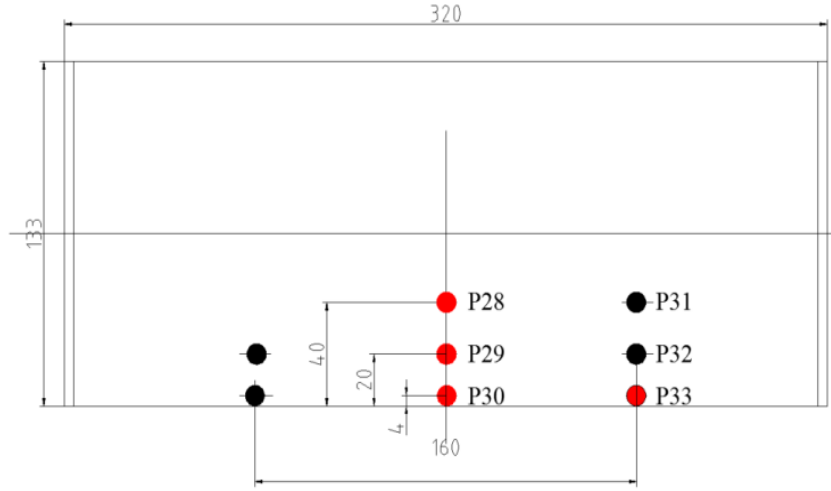

Fig.5 Pressure Monitoring Point on the Baffle

\section{DECK WAVES UNDER REGULAR WAVES}

\section{A. Checking}

In order to the hull movement is closely related to the analysis of the deck wave problem, the accuracy of the method will be verifed. This paper uses Fine/Marine and Starccm+ to analyze the heave and pitch motion of container ships, and compare the difference between the two software calculation results.

Fig.6 and Fig.7 show the heave and pitch motion of the hull at a speed of $18 \mathrm{kn}(\mathrm{Fr}=0.158)$, the wavelength to ship length ratio of $1.1(\lambda / \mathrm{Lpp}=1.1)$ and the amplitude of $0.0555(a=0.0555)$.

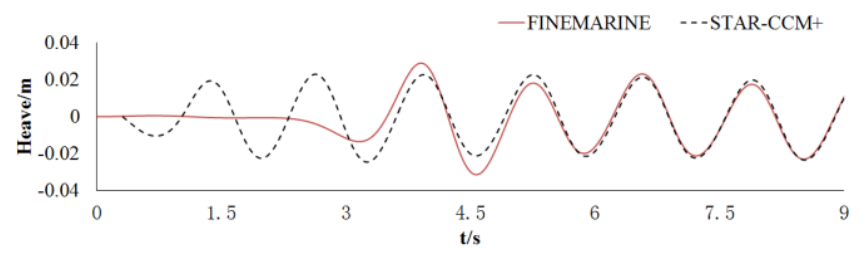

Fig.6 the Heave Motion of Different CFD software

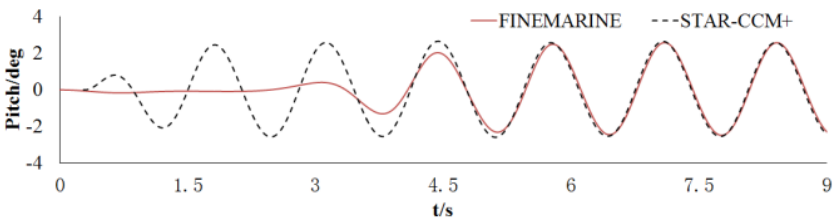

Fig.7 the Pitch Motion of Different CFD Software

It can be seen from figures that the two heaving and pitching time curves obtained by the CFD software agree well, and there are slight differences in the amplitude of the heave. The reason is that CFD software has differences in meshing, calculation settings and solving methods. To some extent, the results obtained by the Fine/Marine software used in this paper have practical reference value.

\section{B. Deck Waves on Different Wave Heights}

In this part, the speed of the vessel is $23 \mathrm{kn}(\mathrm{Fr}=0.202)$, the ratio of wavelength to length $(\lambda / L p p)$ is 1 , wave height $\mathrm{h}=0.098 \mathrm{~m}$ and wave height $\mathrm{h}=0.114 \mathrm{~m}$. The following analysis will be carried out from two aspects of pressure load.

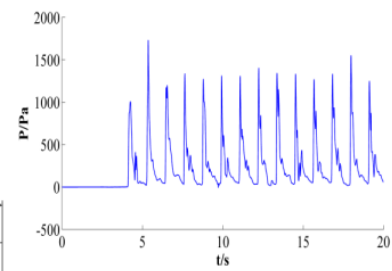

(a) $\mathrm{h}=0.098 \mathrm{~m}(\mathrm{P} 19)$

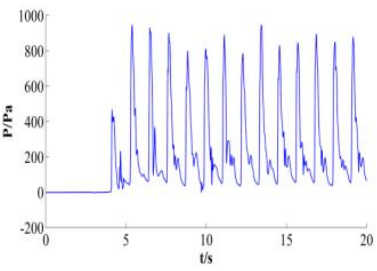

(b) $\mathrm{h}=0.098 \mathrm{~m}(\mathrm{P} 20)$

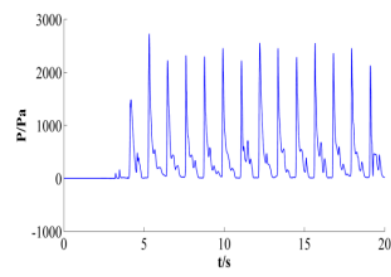

(a)h=0.114m(P19)

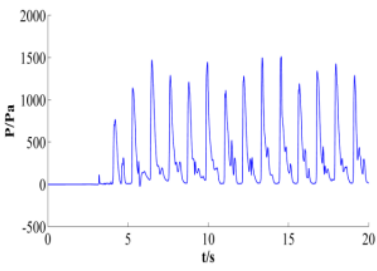

(b)h=0.114m(P20)
Fig.8 Pressure Load of P19 and P20 on Deck 
Two representative pressure points of P19 and P20 on the bow deck were selected for comparative analysis. When the wave heights are different, the higher the wave height of the same measuring point, the greater the pressure on the deck. The reason is that the wave height becomes larger and the wave on the deck is more serious, and the water in the upper wave becomes more so that the deck pressure becomes larger. During the wave on the deck, the waves quickly rushed onto the deck, and the impact of the flowing water on the deck quickly reached its maximum, but as the peak passed, the water gradually flowed out of the deck and the pressure gradually decreased. However, it can be seen from the Fig. 8 that the pressure curve is not always stable. The fluctuation of the pressure peak is due to the fact that after the wave on the deck, the water body of the upper wave does not completely flow out of the deck, and the peak pressure of the deck after the wave is increased again. In general, the pressure on the bow deck is basically stable.

P28 and P29 pressure points are selected on the baffle at the bow deck position for comparative analysis. It can be seen from Fig.9 that when the water hits the baffle, the horizontal impact load on the baffle quickly reaches a peak. However, due to gravity, the rate of rise of the water gradually decreases and begins to fall back, and a second smaller peak is produced at the end of the fallback process. The same wave height, the pressure change curve of different pressure measurement points has a similar overall trend, and the same measurement point corresponds to different wave heights, and the pressure change trend has a large difference. This is because the incident wave height affects the wave level and the water fall speed. In all the monitoring points, the peak of the positive center of the wave shield is the largest. The reason is that after the wave surges onto the deck, it gradually gathers toward the middle and finally gathers at the center of the bottom end of the baffle.

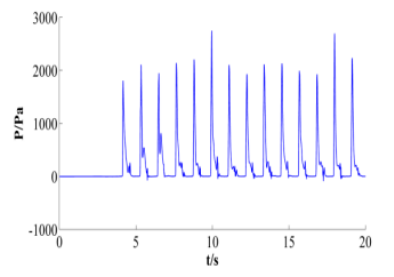

(c) $\mathrm{h}=0.098 \mathrm{~m}(\mathrm{P} 28)$

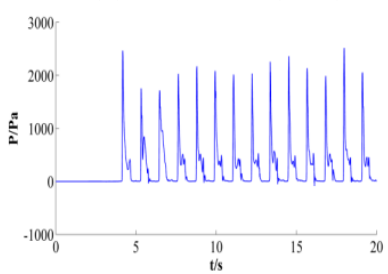

(d)h=0.098m(P29)

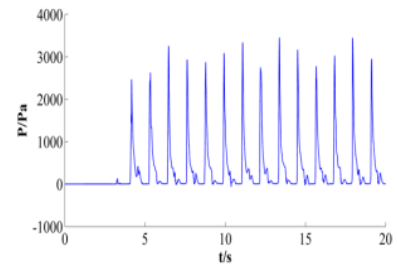

(c) $\mathrm{h}=0.114 \mathrm{~m}(\mathrm{P} 28)$

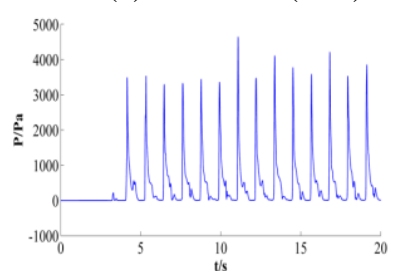

(d) $\mathrm{h}=0.114 \mathrm{~m}(\mathrm{P} 29)$
Fig.9 Pressure Load of P28 and P29 on baffle

The initial pressure jump reflects the process of waves on the deck. Under the specified wave conditions, the height of the pressure point was changed, and it was found that the pressure point at the bottom end of the baffle appeared a pressure jump earlier than the other points. This is because after the front of the wave reaches the bow, the wave energy at the bow begins to accumulate, and the point at the lower end of the baffle first contacts the wave that surges. As the wave energy gradually stabilizes, the middle and upper middle of the baffle begin to wave up, and a stable periodic pressure change occurs. If the height of the pressure point is kept constant and the position of the longitudinal section of the pressure point is changed, the pressure point near the side of the ship will rise in advance, indicating that the wave process starts from the side of the deck and gradually covers the front of the deck. If the position of the pressure point is kept constant and the height of the incident wave is gradually raised, the cycle of the start of the pressure jump is advanced, which reflects the increase of the wave energy from the side, and the rationality of the calculation result is reflected.

\section{Deck Waves at Different Speeds}

The speeds of the container ship are $18 \mathrm{kn}(\mathrm{Fr}=0.158)$ and $23 \mathrm{kn}(\mathrm{Fr}=0.202)$, the ratio of the wavelength to the length of the vessel is 1 and the wave height is $\mathrm{h}=0.114 \mathrm{~m}$. The pressure load will be analyzed below.

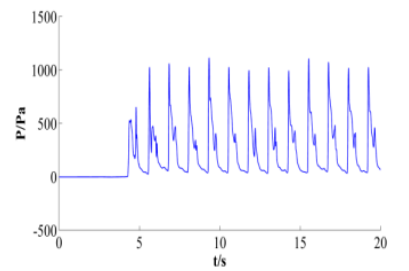

(a) $F r=0.158^{(\mathrm{P} 19)}$

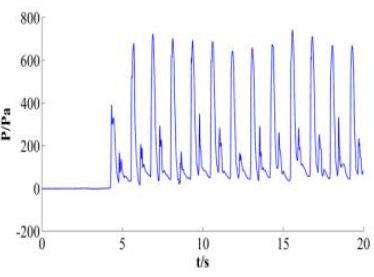

(b) $\operatorname{Fr}=0.158^{(\mathrm{P} 20)}$

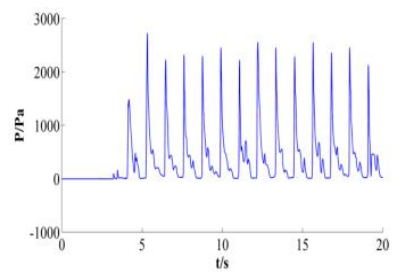

(a) $\operatorname{Fr}=0.202$ (P19)

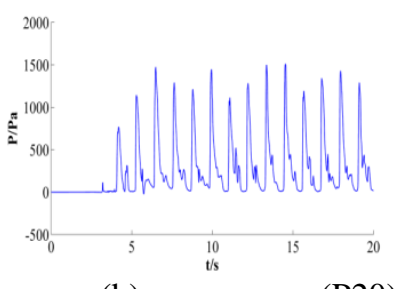

(b) $\mathrm{Fr}=0.202$
Fig.10 Pressure Load of P19 and P20 on Bow Deck

P19 and P20 pressure points on the bow deck are selected for comparative analysis. As can be seen from Fig.10, the greater the speed of the same point, the greater the pressure on the deck. The reason is that the speed of the vessel becomes more serious, and the wave on the deck becomes more serious. The pressure curve measured by the pressure points ( $a$ and b) near the wave shield is as "thin and sharp" as the results measured above, and there is a "double peak" with the characteristics of slamming pressure. During the wave on the deck, the waves quickly rushed onto the deck and hit the windshield and its vicinity directly, causing slamming. The impact of the water on the deck quickly reached its maximum, but as the peak passed, the water gradually flowed out of the deck and the pressure gradually Reduce, so cycle. However, it can be seen from the figure that the pressure curve is not always stable. The fluctuation of the pressure value is because the water on the deck does not completely flow out of the deck after the wave on the deck, which causes the deck pressure value after the wave is increased again, which also reflects $\mathrm{A}$ nonlinear phenomenon in the waves on the deck. In general, there is a certain regularity in the pressure changes after the waves on the bow deck in the regular wave. 


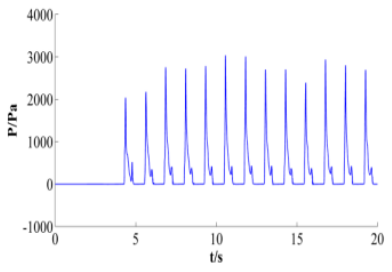

(c) $F r=0.158^{(\mathrm{P} 28)}$

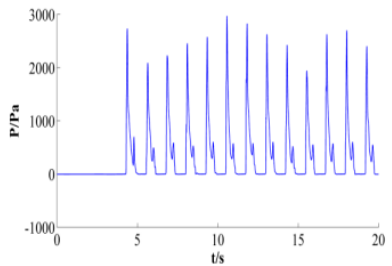

(d) $F r=0.158$ (P29)

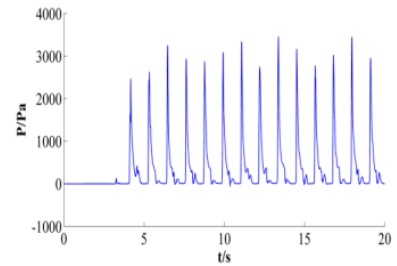

${ }^{(\mathrm{c})}{ }_{F r}=0.202{ }^{(\mathrm{P} 28)}$

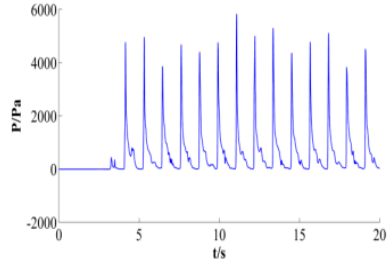

(d) ${ }_{F r}=0.202^{(\mathrm{P} 29)}$
Fig.11 Pressure Load of P28 and P29 on baffle

Fig.11 is a comparison of the pressure-time curves of the various pressure points on the baffle at different speeds. Two representative pressure points P28 and P29 on the on the baffle of the bow deck were selected for comparative analysis.

It can be seen from Fig.11 that the pressure change curve measured by all the monitoring points on the baffle is "thin and sharp" and the "double peak" slamming pressure characteristics are caused when the water hits the baffle. The horizontal slamming load on the baffle quickly peaks. However, due to gravity, the rate of rise of the water body gradually decreases and begins to fall back, and a second smaller peak is produced at the end of the fallback process. At the same speed, the pressure change curve of different pressure points has a similar overall trend, while the pressure change trend of the same point corresponding to different speeds is different. This is because the speed of the ship affects the wave level and the water body fall speed. In all the measuring points, the pressure at the center of the center of the baffle is the largest. The reason is that after the wave surges onto the deck, it begins to gradually gather toward the center and finally gathers at the center of the bottom end of the baffle.

In the pressure curve of the Friedel number $\mathrm{Fr}=0.202$, it can also be seen that a small peak appears around $3.5 \mathrm{~s}$, and when the Friedel number $\mathrm{Fr}=0.158$, there is no such small peak, the reason is as described above.

\section{CONCLUTION}

This paper introduces the background and significance of the waves on the deck firstly, and summarizes and reviews the simulation method of the waves on the deck. It describes the research methods and latest research progress of the waves on the deck, and then summarizes the basic research methods for the research.

1. Create a viscous numerical tank and select the micro amplitude theory to simulate a regular wave. To verify the accuracy of Fine/Marine calculations on the deck, use the CFD software Starcem+ to compare with the results of Fine/Marine to analyze the movement of the container in the waves.

2. In order to study the wave conditions on the deck, the pressure loads on the bow deck and the baffle are analyzed from different wave heights and different speeds, and the pressure load changes at different pressure points and the reasons for the changes are analyzed. It was found that the deck waves had a considerable degree of pressure impact on the bow deck and the baffle, which provided a certain reference value for ensuring the strength of the bow deck and protecting the equipment on the deck during the construction of the actual ship.

\section{REFERENCES}

[1] Newton R. Wetness related to freeboard and flare. Trans. RINA. 1960 102:49.

[2] Nielsen K.B. Stefan M. Numerical prediction of green water incidents. Ocean Engineering, 2004, 31(3-4):363-399.

[3] Hu C, Kashiwagi M, Kitadai A. Numerical simulation of strongly nonlinear wave-body interactions with experimental validation. Proceedings of The 3rd Asia-Pacific Workshop on Marine Hydrodynamics, Shanghai, China, 2006, 232-236.

[4] Rik, Wemmenhove, Roel, Luppes, EP Arthur. Numerical Simulation of Sloshing in LNG Tanks with a Compressible Two-Phase Model. Asme International Conference on Offshore, 2007, 871-879.

[5] Bowen Shi, Zhengjiang Liu, Ming Wu. Numerical simulation of deck waves based on current wave spectrum [J]. Journal of Dalian Maritime University: Natural science edition, 2016(3):31-36.

[6] Guanghua He, Limin Cheng, Jiadong Wang. Strong nonlinear time domain simulation of ship motion in waves [J]. Journal of Harbin Institute of Technology, 2017(4). 